\title{
Capturing the Atomic Coordinates of Surface and Subsurface Structure in 4D with Atomic Electron Tomography
}

Jihan Zhou ${ }^{1}$, Yongsoo Yang ${ }^{2}$, Yao Yang ${ }^{1}$, Colin Ophus ${ }^{3}$, Fan Sun ${ }^{4}$, Andreas Schmid ${ }^{3}$, Hao Zeng ${ }^{4}$, Peter Ercius $^{3}$ and Jianwei Miao ${ }^{1}$

${ }^{1}$ University of California-Los Angeles, Los Angeles, California, United States, ${ }^{2}$ Korea Advanced Institute of Science and Technology, Daejeon, Ch'ungch'ong-bukto, Republic of Korea, ${ }^{3}$ Lawrence Berkeley National Laboratory, Berkeley, California, United States, ${ }^{4}$ University of Buffalo, Buffalo, New York, United States

The atomic arrangement of surface/subsurface structure is critical for many applications ranging from catalysis and electronics to biosensing and targeted delivery [1]. Currently, there are several surface structure analysis methods, including electron scattering, X-ray scattering, spectroscopic methods and scanning probe microscopy such as atomic force microscopy (AFM) and scanning tunneling microscopy (STM) [2]. AFM and STM can provide a real-space mapping of surface atoms with sub-atomic resolution. However, the 3D atomic coordinates of the surface and especially subsurface cannot be easily accessed by those methods. Over the past few years, in a combination of the state-of-the-art electron microscopy, powerful 3D reconstruction and atom tracing algorithms, a groundbreaking method, termed atomic electron tomography (AET) [3], has been developed to determine the 3D atomic structures, including surface and subsurface atomic structures, of nanomaterials without the assumption of crystallinity [4-7]. More recently, we established a state-of-art data acquisition method to add a new dimension (time) to AET (i.e. 4D-AET) to study early stage nucleation dynamics in solid-solid phase transitions [8]. Using an iron-platinum (FePt) nanoparticle as a model system, we advanced AET to capture how the surface atoms rearrange during early nucleation at $4 \mathrm{D}$ atomic resolution. By annealing the same $\mathrm{FePt}$ nanoparticle at $520{ }^{\circ} \mathrm{C}$ in vacuum at different annealing times, we obtained a sequential series of three models of the same particle with an accumulated annealing time of 9, 16 and 26 minutes, respectively. The surface and subsurface atomic positions and chemical ordering information with a 3D precision of 26 picometers is shown in Fig. 1. All the nuclei are situated on the surface or subsurface. Most nucleation sites are located on the facets, edges or corners (Fig. 2). This experiment adds a new dimension (time) to AET, allowing the observation of time evolution of structure in materials in four atomic dimensions, which is currently not accessible by any other experimental methods. We anticipate that AET will play a key role in helping to solve some fundamental problems in materials science, nanoscience, condensed-matter physics, and chemistry [9]. 

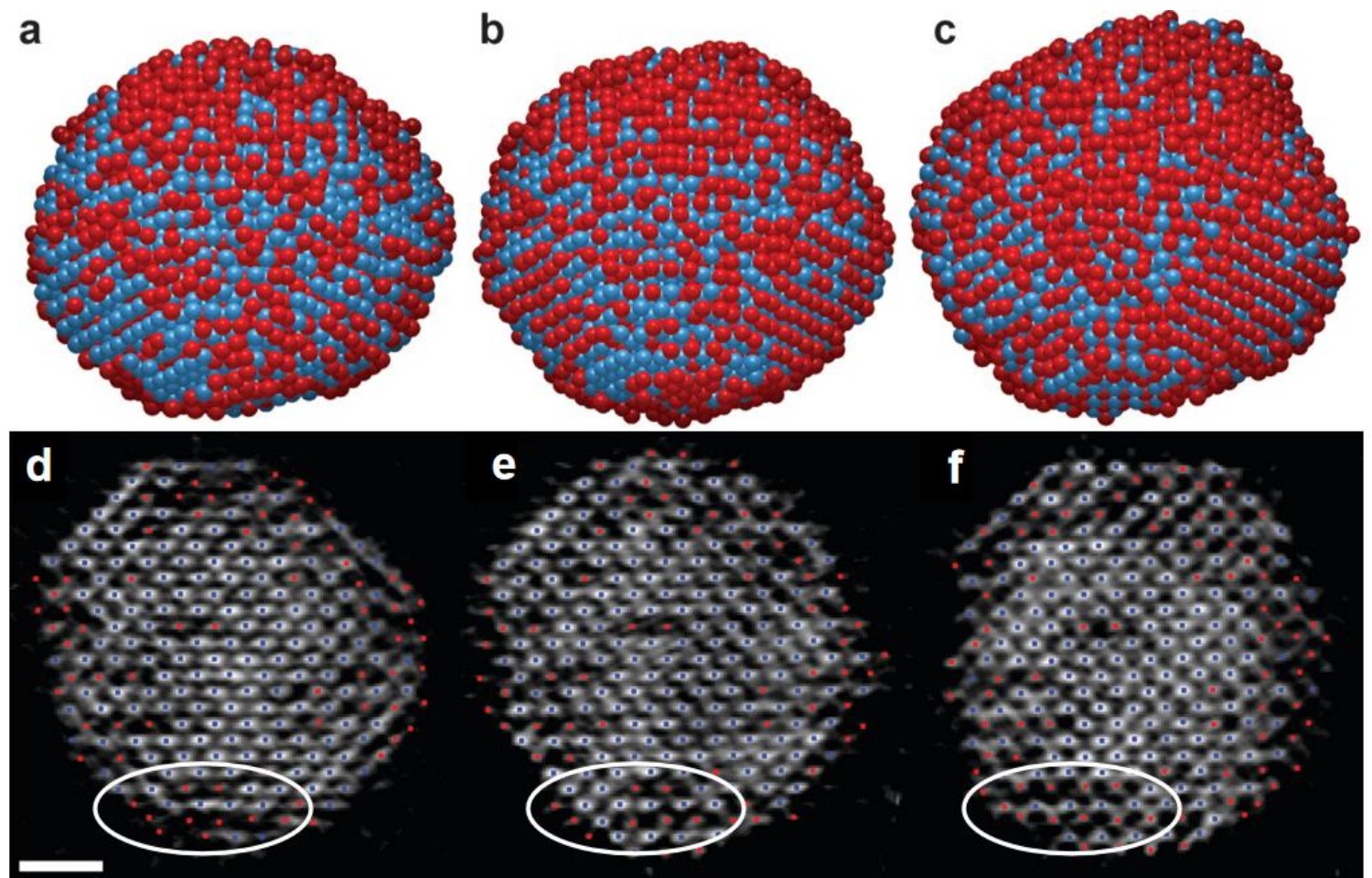

Figure 1. Capturing surface/subsurface atomic motion and nucleation dynamics with 4D-AET. a-c, 3D atomic models ( $\mathrm{Fe}$ in red and $\mathrm{Pt}$ in blue) of an FePt nanoparticle annealed at $520 \mathrm{oC}$ with an accumulated annealing time of $9 \mathrm{~min}$ (a), $16 \mathrm{~min}$ (b), and $26 \mathrm{~min}$ (c), respectively. d-f, The same internal atomic layer of the nanoparticle along the [010] direction at the three annealing times.(Fe in red and Pt in blue; Scale bar, $1 \mathrm{~nm})$.
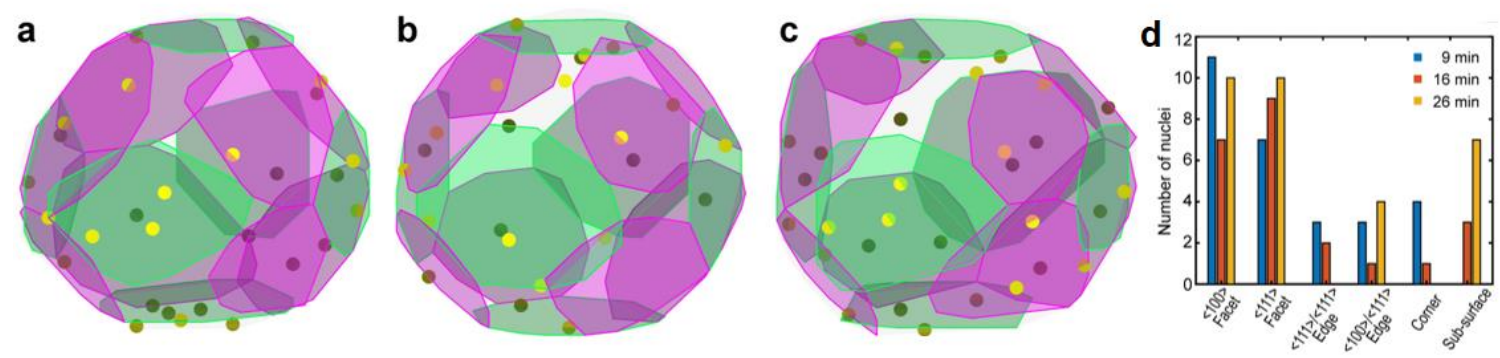

Figure 2. Revealing the heterogeneous nucleation sites on the surface and subsurface of the particle. a-c, The distribution of the nucleation sites (circular dots) in the particle with an accumulated annealing time of 9, 16 and 26 minutes, respectively, where the lighter colored dots are closer to the front side and the darker dots are closer to the back side of the nanoparticle. The and facets are in magenta and green, respectively. $\mathrm{d}$, The histogram of the nucleation site distribution in the particle, where most nucleation sites are located on the facets, edges or corners.

\section{References}

[1] A. Kelly, K. Knowles, Crystallography and Crystal Defects (Wiley, 2012).

[2] A. Nilsson, L. G.M. Pettersson \& J. K. Nørskov, Chemical Bonding at Surfaces and Interfaces, (Elsevier, 2008).

[3] J. Miao et al., Science 353, (2016), p. aaf2157.

[4] M. C. Scott et al., Nature 483, (2012), p. 444-447. 
[5] C. C. Chen et al., Nature 496, (2013), p. 74-77.

[6] R. Xu et al., Nat. Mater. 14 (2015), p. 1099-1103.

[7] Y. Yang et al., Nature 542 (2017), p. 75-79.

[8] J. Zhou et al., Nature 570, (2019), p.500-503.

[9] This work was primarily supported by STROBE: A National Science Foundation Science \& Technology Center under Grant No. DMR 1548924 and was also supported by the Office of Basic Energy Sciences of the US DOE (DE-SC0010378) and the NSF DMREF program (DMR-1437263). The experiment was performed at the Molecular Foundry, which is supported by the Office of Science, Office of Basic Energy Sciences of the U.S. DOE under Contract No. DE-AC02-05CH11231. 